\title{
Fatal bupivacaine overdose through intrathecally positioned epidural catheter
}

\author{
Lina Davies Forsman¹, MD, Mats Öström¹, MD PhD, Mikael Svanström², MD, Anders Eriksson1*, MD PhD
}

'Section of Forensic Medicine, Dept of Community Medicine and Rehabilitation, Umeå University, PO Box 7642, SE-907 12 Umeå, Sweden

${ }^{2}$ Dept of Surgical and Perioperative Sciences, Umeå University, SE-901 85 Umeå, Sweden

* anders.eriksson@rmv.se

\begin{abstract}
We describe a fatality due to an intrathecally positioned epidural catheter and an infusion rate of bupivacaine set 10 times higher than planned. The undetected misplacement, despite safety routines, is discussed along with the toxicological findings and new information on the intrathecal distribution of bupivacaine.

From a clinical point of view, the human factor, in combination with an indistinct decimal point on the pump, was considered as the reason for the unfortunate overdose. In continuous epidural infusion of local anesthetics, the importance of guidelines and informed staff in managing complications of epidural lumbar infusion as well as careful monitoring of the vital functions is essential. Guidelines are also vital during the procedure of insertion of epidural catheters. When using combined spinal and epidural anaesthesia, we believe that an epidural catheter should be inserted, and its position tested, prior to spinal anesthesia.

The case also illustrates the need of innovative investigation techniques to confirm the suspicion of unusual manifestations of inadvertent drug effects. Segmental analysis, together with analyses in a control case, enabled us to elucidate the high and varying tissue concentrations in the central nervous system.
\end{abstract}

Keywords:

Guidelines, Spinal cord, Toxicology

\section{INTRODUCTION}

Misplacement of an epidural catheter and subsequent injection of local anaesthetic into the subarachnoid space may have disastrous consequences. Hence, careful measures are taken to ensure the correct placement of the catheter, and a test dose is generally administered. The test dose generally contains adrenaline, in order to produce palpitations to reveal a possible intravenous placement. An intrathecal injection of the test dose usually produces the onset of spinal anaesthesia within minutes. Negative aspiration of cerebrospinal fluid (CSF) and a negative response to the test dose is, however, not proof of a correct position [1].

The dose of a local anaesthetic at the lumbar epidural level is approximately 5-7 times higher than the corresponding intrathecal dose. If such an excessive dose is deposited intrathecally it will result in a higher level of blockage, risking respiratory depression and circulatory collapse, or even a total spinal anaesthesia with sudden unconsciousness, severe hypotension, and respiratory depression [2]. We describe here a case of an intrathecally positioned epidural catheter with fatal overdose of bupivacaine. The undetected misplacement, despite safety routines, is discussed along with the toxicological findings and new information on the distribution of bupivacaine.

\section{CASE REPORT}

A 71-year-old woman with arterial insufficiency in the right leg was scheduled for vascular surgery. Her body weight was $60 \mathrm{~kg}$, height 163 $\mathrm{cm}$, and her medical history included hypertension, cardiac hypertrophy, diabetes type 2, three femoral by-pass operations and amputation of the left leg.

Preoperative angiography revealed a total occlusion of a vein graft in the right leg, and a combined spinal and epidural anaesthesia was applied. The spinal anaesthesia was administered with a 22 gauge needle at the L3-L4 level using $16.25 \mathrm{mg}$ of bupivacaine $(0.5 \%)$ and $0.3 \mathrm{mg}$ morphine, producing a Th5 sensory blockade. An epidural catheter was subsequently inserted with a 16 gauge needle aimed through the L2-L3 interspace for post-operative pain relief. The exploration was uneventful.

Postoperatively at $3 \mathrm{pm}$, a test dose of $12.5 \mathrm{mg}$ of bupivacaine $0.25 \%$ was injected through the epidural catheter. At $3.35 \mathrm{pm}$, an epidural infusion of $4 \mathrm{ml}$ of bupivacaine $0.25 \%$ per hour was initiated. At $4.30 \mathrm{pm}$, after administration of $22.5 \mathrm{mg}$, the patient became hypotensive, and the infusion was stopped. The blood pressure was normalized with intravenous acetated Ringer's solution. A new epidural infusion rate of $2 \mathrm{ml}$ per hour was activated at $5.40 \mathrm{pm}$, the patient remained stable and was transferred to a regular ward. At $11.30 \mathrm{pm}$ she was asleep, although vital signs were not controlled. At midnight she was found pulseless and resuscitation was unsuccessful. It was revealed that the infusion rate had been set to $20 \mathrm{ml}$ per hour, i.e. ten times higher than prescribed. The event was reported to the police and to the National Board of Health and Welfare, and a medico-legal autopsy was performed.

\section{Medico-legal autopsy}

The epidural catheter, fixed by adhesive tape, had been placed in the midline at the L1-L2 level. Further, the tip of the catheter had penetrated the dura 
and was positioned $4.3 \mathrm{~cm}$ caudally in the spinal canal. A small epidural hemorrhage was seen around the L1-L2 insertion site, as well as at the T12-L1 level. Advanced general and coronary atherosclerosis, and diffuse myocardial fibrosis were other significant findings.

The spinal cord was sectioned into $5 \mathrm{~cm}$ long segments $(n=8)$, and a $3 \mathrm{~cm}$ caudal segment which consisted solely of nerve fibers. The pons and the medulla oblongata were sectioned into 4 and 2 pieces, respectively. The spinal cord, pons and medulla oblongata were frozen at $-70^{\circ} \mathrm{C}$, pending toxicological analyses.

\section{Toxicological analyses}

Toxicological analysis in femoral blood was performed according to Druid and Holmgren [3] using gas chromatography with nitrogen phosphorous detection. Two extracts, one basic and one neutral, were analyzed on two different columns to identify and quantify pharmaceutical drugs.

Identification and quantification of pharmaceutical drugs in tissues were performed by a method modified from Eklund et al [4], also using two extracts and dual columns as described above.

Femoral blood contained $1.5 \mu \mathrm{g}$ bupivacaine, $0.3 \mu \mathrm{g}$ dextropropoxyphene, and $1 \mu \mathrm{g}$ paracetamol per gram. The concentration of bupivacaine in each segment of the spinal cord, the pons and the medulla oblongata is shown in Figure 1.

\section{Medical assessment}

The National Board of Health and Welfare acquitted the nurse responsible for the overdose, and the anaesthesiologist for the incorrect positioning of the catheter. The lack of guidelines for the management of continuous epidural anaesthesia at the hospital was thought to have been a contributory factor, and such guidelines were requested.

The three infusion pumps used were all of the same brand with no technical failures found upon tests.

\section{DISCUSSION}

The inadvertent intrathecal position of the epidural catheter is intriguing. The question arises why there were no physical reactions to the test dose and to the subsequent infusion, indicating the incorrect position of the catheter.

\section{Test dose and the catheter position}

Negative aspiration of CSF and a negative test dose response is not proof of correct placement of the catheter $[1,5]$, but in case of misplacement, the subsequent infusion usually causes extension of the block and hypotension. A drop in blood pressure did indeed occur, but not until one hour after the start of the infusion, and was interpreted as a sign of the general instability of the patient's circulatory system. Possibly, the lack of response to the test dose was due to the spinal anaesthesia preceding the test dose [6]. For this reason, testing the localization of the epidural catheter localization prior to the administration of the spinal block is preferable [7]. In this case, however, activation of the epidural catheter was made post-operatively, possibly before the spinal anaesthesia had ceased.

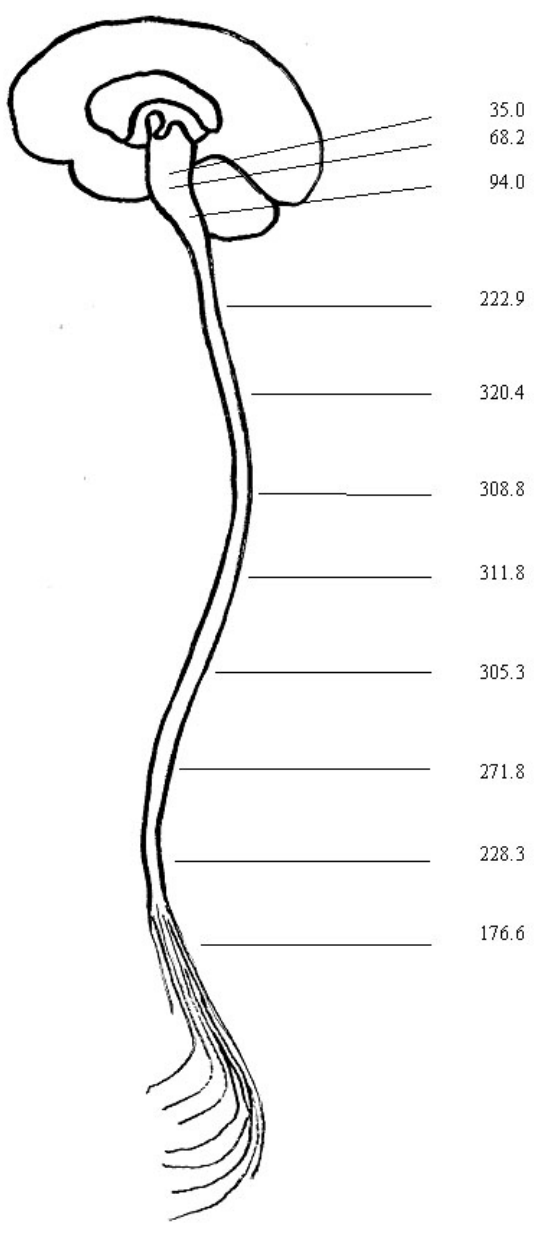

Figure 1. Bupivacaine concentration ( $\mu \mathrm{g} / \mathrm{g}$ tissue) in the pons, medulla oblongata, and spinal cord. The concentrations in the upper pons, the lower pons, and the medulla oblongata, represent the mean of measurements left and right.

To explain the autopsy findings, the risk of catheter migration into the subarachnoid space must be considered $[1,8]$. This phenomenon in combined spinal and epidural anaesthesia (CSE) is thought to be due to the epidural catheter passing through the dural hole previously made by the spinal needle [9]. In cadavers it was, however, not possible to force an (18 gauge) epidural catheter through a dural tear (made by a 25-gauge spinal needle), concluding that the risk of catheter migration during CSE is very small [9].

In the present case, the spinal anaesthesia was administered at the L3-L4 level, and the epidural catheter was intended to pass the L2-L3 interspace but was actually inserted one interspace higher. These findings make it impossible for the catheter to pass through the dural tear made by the spinal needle. Further, catheter migration in the present case seems remotely plausible, since the catheter was safely secured onto the skin, and since a considerable part of the catheter was positioned intrathecally.

Inadvertent penetration of the epidural catheter per se has been reported to occur [10], although with difficulty [11]. Dural puncture while inserting the epidural catheter, disguised by residual spinal anaesthesia, seems to be the explanation of the findings in our case. Increased sympathetic block due to intrathecal injection may have produced the described hypotensive event in the early post-operative course. 


\section{Bupivacaine toxicity and interpretation of toxicological findings}

The incorrect dose rate was probably set at the arrival at the ward, when the infusion pumps were changed. Consequently, the patient received $100 \mathrm{mg}$ of bupivacaine in a $2 \mathrm{~h}$ period $(1.7 \mathrm{mg} / \mathrm{kg})$, or $144 \mathrm{mg}$ in a $9 \mathrm{~h}$ period.

Bupivacaine is more cardiotoxic than other commonly used local anaesthetics [12], with a narrow gap between convulsant and lethal doses in experimental studies [13]. The cardiotoxic effects may be due to direct effect on the myocardium or mediated through the central nervous system [14].

Although bolus intravenous injections do not fully compare to the present case where blood concentrations changed more slowly, venous plasma levels of $1.6-4.5 \mu \mathrm{g} / \mathrm{g}$ (corresponding to $1-3 \mu \mathrm{g} / \mathrm{g}$ whole blood [15]) did not cause severe toxic symptoms in human volunteers [15]. On the other hand, a whole blood concentration of $1.8 \mu \mathrm{g} / \mathrm{g}$ in a 71-year old man was associated with convulsions, bradycardia and loss of conciousness [16].

Consequently, the importance of the blood concentration $(1.5 \mu \mathrm{g} / \mathrm{g})$ in our case remains obscure. Even though systemic effects of the drug cannot be ruled out, we favor that the more important effects were mediated through the intrathecal route of administration.

We have found no previous publication on CNS concentrations of bupivacaine in a fatal overdose, which makes the levels found (Figure 1) hard to interpret. In order to shed some light on this problem, we analyzed in a similar manner the spinal cord of a 72-year-old man who died from severe ischemic heart disease with acute myocardial infarction while receiving continuous epidural infusion of bupivacaine for pain relief in metastatic cancer. Analyses showed a mean concentration of bupivacaine of $8.1 \mu \mathrm{g} / \mathrm{g}$ spinal cord and $0.2 \mu \mathrm{g} / \mathrm{g}$ femoral vein blood, clearly indicating that the CNS concentrations found in our fatality were very high.

\section{ACKNOWLEDGEMENT}

We are indebted to Dr Robert Kronstrand, Dept of Forensic Toxicology, Linköping, for valuable cooperation.

\section{REFERENCES}

[1] Troop M. Negative aspiration for cerebral fluid does not assure proper placement of epidural catheter. AANA J. 1992, 60, 301-303

[2] Collier C. Total spinal or massive epidural block? [letter] Anaesth. Intensive Care 1998, 10, 92

[3] Druid $\mathrm{H}$, Holmgren P. A compilation of fatal and control concentrations of drugs in postmortem femoral blood. J. Forensic. Sci. 1997, 42, 79-87

[4] Eklund A, Jonsson J, Schuberth J. A procedure for simulataneous screening and quantification of basic drugs in liver, utilizing capillary gas chromatography and nitrogen sensitive detection. J. Analyt. Toxicol. 1983, 7, 24-28

[5] Galet A, Fleyfel M, Beague D, Vansteenberghe F. Accidental spinal anaesthesia in obstetrics: limits of an epidural testdose. Ann. Fr. Anesth. 1992, 11, 377-380

[6] Suzuki N, Koganermaru M, Onizuka S, Takasaki M. Dural puncture with a 26-gauge spinal needle affects spread of epidural anaesthesia. Anesth. Analg. 1996, 82, 1040-1042

[7] Wilhelm S, Standl T, Burmeister M, Kessler G, Schulte am Esch J. Comparison of continuous spinal with combined spinal epidural anesthesia using plain bupivacaine 0,5\% in trauma patients. Anesth. Analg. 1997, 85, 69-74

[8] Park R. A migrating epidural cannula [letter]. Anaesthesia 1984, 39, 289

[9] Holmström B, Rawal N, Axelsson K, Nydahl P-H. Risk of catheter migration during combined spinal epidural block: Percutaneous epiduroscopy study. Anesth Analg. 1995, 80, 747-753
[10] Lin H-Y, Wu H-S, Peng T-H, Yeh YJ, Cheng IC, Lin IS, Liu CH. Pneumocephalus and respiratory depression after accidental dural puncture during epidural analgesia - a case report. Acta Anaesthesiol Sin. 1997, 35, 119-123

[11] Hardy PAJ. Can epidural catheters penetrate dura mater? An anatomical study. Anaesthesia 1986, 41, 1146-1147

[12] Naguib M, Magboul MMA, Samarkandi AH, Attia M. Adverse effects and drug interactions associated with local and regional anaesthesia. Drug Safety 1998, 18, 221-250

[13] de Jong RH, Bonin JD. Deaths from local anesthetic-induced convulsions in mice Anesth. Analg. 1980, 59, 401-405

[14] Thomas RD, Behbehani MM, Coyle DE, Denson DD. Cardiovascular toxicity of local anesthetics: An alternate hypothesis. Anesth. Analg. 1986, 65, 444-450

[15] Reynolds F. A comparison of the potential toxicity of bupivacaine, lignocaine and mepivacaine during epidural blockade for surgery. Brit. J. Anaesth. 1971, 43, 567572

[16] Rosenberg PH, Kalso EA, Tuominen MK, Lindén HB. Acute bupivacaine toxicity as a result of venous leakage under the tourniquet cuff during a Bier block. Anesthesiology 1983, 58, 95-98 